BMJ Open

Diabetes

Research

\& Care

\title{
Implementation of routine foot check in patients with diabetes on hemodialysis: associations with outcomes
}

\author{
Andreja Marn Pernat,, ${ }^{1,2}$ Vanja Peršičc, ${ }^{1,2}$ Len Usvyat, ${ }^{2,3}$ Lynn Saunders, ${ }^{2}$ \\ John Rogus, ${ }^{3}$ Franklin W Maddux, ${ }^{3}$ Eduardo Lacson Jr, ${ }^{4}$ Peter Kotanko ${ }^{2,5}$
}

To cite: Marn Pernat $A$, Peršič V, Usvyat L, et al. Implementation of routine foot check in patients with diabetes on hemodialysis: associations with outcomes. BMJ Open Diabetes Research and Care 2016;4:e00158. doi:10.1136/bmjdrc-2015000158

Received 4 October 2015 Revised 17 January 2016 Accepted 26 January 2016

CrossMark

${ }^{1}$ University Medical Center Ljubljana, Ljubljana, Slovenia ${ }^{2}$ Renal Research Institute, New York, New York, USA ${ }^{3}$ Fresenius Medical Care North America, Waltham, Massachusetts, USA

${ }^{4}$ Tufts University School of Medicine, Boston,

Massachusetts, USA

${ }^{5}$ Icahn School of Medicine at Mount Sinai, New York, New York, USA

Correspondence to Dr Andreja Marn Pernat; andreja.marn@kclj.si

\section{ABSTRACT}

Objective: Patients with diabetes are at increased risk of foot ulcers, which may result in limb amputations. While regular foot care prevents ulcerations and amputation in those patients with diabetes not on dialysis, evidence is limited in diabetic hemodialysis patients. We investigated the association between the implementation of a routine foot check program in diabetic incident hemodialysis patients, and major lower limb amputations.

Methods: In 1/2008, monthly intradialytic foot checks were implemented as part of standard clinic care in all Fresenius Medical Care North America hemodialysis facilities. Patients with diabetes who initiated hemodialysis between 1/2004 and 12/2007 constituted the preimplementation cohort, and patients starting hemodialysis between 1/2008 and 12/2011 comprised the postimplementation cohort. In addition, we conducted a sensitivity analysis where we excluded patients from the clinics with $<10$ patients in the postimplementation period and where percent difference in patient with diabetes number between postimplementation and preimplementation period was $<20 \%$. We compared lower limb amputation rates employing Poisson regression models with offset of exposure time in these two cohorts.

Results: We studied 35513 patients in the preimplementation and 25779 patients in the postimplementation cohort. In the postimplementation cohort, amputation rate decreased by $17 \%(p=0.0034)$. The major lower limb amputation rate was 1.30 per 100 patient years in preimplementation and 1.07 in postimplementation cohort. These beneficial results were corroborated in the multivariate analysis $(p=0.0175)$ and were even more pronounced in the sensitivity analysis $(p=0.0083)$.

Conclusion: Monthly foot checks are associated with reduction of major lower limb amputations in diabetic incident hemodialysis patients.

\section{INTRODUCTION}

Patients with diabetes on hemodialysis are at increased risk of foot complications, which may eventually result in lower extremity amputations, resulting in the loss of parts of toes, feet or even legs. Recurrence of

\section{Key messages}

- Optimizing foot care with routine monthly intradialytic foot checks was implemented for all diabetic hemodialysis patients in a large US-based hemodialysis network in 2008.

- This observational study reports the association between the implementation of a routine foot check program in diabetic incident hemodialysis patients, and major limb amputations.

- Implementation of the monthly foot check program was associated with a significant $17 \%$ lower rate of major lower limb amputations, when compared with the preimplementation period.

these problems in the contralateral limb is frequent. $^{12}$

Foot complications are more prevalent in patients with long-standing diabetes and endstage renal disease compared with those without end-stage renal disease. Hill et al previously demonstrated risk of foot complications defined as current ulcer, infection, gangrene or amputation in this group of patients. Foot complications were greater in patients with diabetes on hemodialysis $(25 \%)$ than in patients with diabetes not on dialysis (10\%). A prevalence of lower limb amputations in diabetic hemodialysis patients was $11 \%$ compared with $4 \%$ of patients with diabetes not on dialysis. ${ }^{3}$ Ndip et $a t^{4}$ reported that in patient with diabetes and chronic kidney disease the prevalence of foot ulceration was five times higher in patients on hemodialysis compared with predialysis patients (chronic kidney disease stages 4 or 5). The dialysis group with 150 patients with diabetes had higher rates of foot ulcerations and amputations, with estimated cumulative incidence rates of 210 and 58 per 1000 person-years, respectively, when compared with 150 patients with diabetes with a history of foot ulceration. ${ }^{5}$

Foot ulcers precede $84 \%$ of amputations, ${ }^{6}$ and half of these amputations are in patients 
with diabetes. ${ }^{7}$ In 1994, the overall amputation rate for patients on hemodialysis with renal failure secondary to diabetes was 13.8 events/100 persons per year versus 4.9 events/100 persons per year for patients with end-stage renal disease unrelated to diabetes. The non-traumatic amputation rate among patients with diabetes on hemodialysis in the USA was 10 times as great as among the diabetic population at large, ${ }^{1}$ suggesting that diabetes is a major risk factor for lower limb amputation in hemodialysis patients. $^{8}$

Amputations are associated with reduced quality of life, morbidity, and increased mortality. Following amputation, more than one-third of the patients are left unable to care for themselves; above ankle amputation is associated with a perioperative mortality of $20 \% .^{1} 9$ Two-thirds of the patients died within 2 years after the first amputation. ${ }^{1}$ Recently, a 5-year observational study demonstrated increased risk of mortality in hemodialysis patients with foot ulcerations. ${ }^{10}$

As foot ulcers are a significant risk factor for limb loss, prevention, and timely diagnosis and treatment, may translate into a reduced amputation rate. In nonhemodialysis patients, several approaches have been shown to prevent lower limb ulceration and amputation; however, information is limited on patients with diabetes who are on hemodialysis. Two small, single-center studies have shown foot care extending to the hemodialysis setting was beneficial in reducing amputation rates. An intensive podiatric and orthotic service was instituted for New Zealand Mäori people with diabetes after they began dialysis. The lower extremity amputation rate fell from 14 patients for the previous 2 years to 2 per year. ${ }^{11}$ When foot care was extended to all diabetic Native Americans who were receiving dialysis in one local facility, amputation rates decreased from 15 per 1000 to 7 per 1000 diabetic person-years in the period from 1997 to $1999 .^{12} 13$ The KDOQI guidelines recommend screening including physical examination with assessment of arterial pulse and skin integrity, at the time of dialysis initiation for patients with chronic kidney disease on chronic renal replacement therapy. ${ }^{14}{ }^{15}$ As dialysis patients often lose contact with care outside the dialysis setting, preventive strategies as a part of routine dialysis care may be key for decreasing amputation rates.

In order to improve foot care in chronic hemodialysis patients, monthly intradialytic foot checks performed by dialysis nurses were implemented in all Fresenius Medical Care North America clinics in January 2008. However, current evidence is weak regarding whether regular surveillance and timely recognition of foot lesions in chronic diabetic hemodialysis patients is effective in reducing the risk of amputation and its sequelae, including mortality.

The aim of this study was to investigate the association between foot checks in diabetic hemodialysis patients and rate of lower limb amputations. To explore this relationship, we compared outcomes in incident hemodialysis patients with diabetes before and after the implementation of a comprehensive foot check program in January 2008.

\section{METHODS}

Participants

This observational study was approved by the Institutional Review Board of Beth Israel Medical Center, New York. We performed a retrospective chart review on Fresenius Medical Care North America in-center diabetic hemodialysis patients from 934 dialysis centers. Data were collected from 1 January 2004 to 31 December 2011. The study included incident patients aged older than 18 years with diabetes mellitus who started chronic hemodialysis before the implementation of a foot check program (between January 2004 and December 2007; preimplementation cohort) and thereafter (between January 2008 and December 2011; postimplementation cohort). Patients were required to have at least 13 in-center treatments before being included in the study. We studied patients in clinics performing foot check on a regular basis, defined as more than one foot check per 15 hemodialysis treatments in the postimplementation period. The same clinics were studied in the preimplementation period to reduce the potential for bias.

Amputations were noted between January 2004 and December 2011. Amputation information was obtained through hospitalization records. A major amputation was considered as the presence of a non-traumatic lowerextremity amputation. Lower-extremity amputation was defined as the complete loss in the transverse anatomical plane of any part of the lower limb through or proximal to the ankle joint (International Classification of Diseases, Ninth Revision (ICD-9) codes 841.2; 841.3; 841.4; 841.5; 841.6; 841.7).

\section{Foot check protocol}

In order to improve foot care, monthly intradialytic foot checks by dialysis nurses were implemented as part of standard clinic care in Fresenius Medical Care North America clinics in January of 2008 ('foot check program implementation date'). Within the first 30 days of admission into the dialysis center, an initial foot check was performed. Foot checks were conducted according to the following standard operation procedure: First, history from the patient in regard to any foot-related issues was taken and documented on the initial foot check form. The history included any lower limb pain, history of past or present foot ulcers, amputations, or deformities. Information was comprehensively gathered about podiatry or wound care clinics the patient had visited in the past and about any future appointments the patient had possibly scheduled. After this data had been obtained, physical assessments of the lower extremities started, and continued monthly thereafter. Prior to removing shoes from the patient's feet, an examination was performed 
on the shoes to check for proper fit and appropriateness, and of the inside of the shoe to identify possible pressure points. The socks were examined for holes that could cause friction leading to a blister. The color, temperature, and skin integrity of each foot were noted. The nurse examined the foot anterior and posterior aspects including nails, visually inspecting for any noticeable areas of skin breakdown or reddened areas. Pedal pulses, both the dorsalis pedis and posterior tibial pulses, were palpated and noted if present, diminished, or absent. Foot sensory levels were assessed with a 5.07 monofilament. If any new wounds were observed, the physician was notified and the first available appointment with the wound care center was scheduled. If the patient needed nail trimming, then an appointment with the podiatry clinic was scheduled. Referrals were made to the orthopedic clinic, if the need for diabetic shoes was noted. Another important role of the nurse during the foot check process, was to educate the patient. First, the patient's basic knowledge level was assessed followed by simple education during the actual process of checking the feet. Patients were reminded to check their feet every day. They were instructed to wear appropriate shoes and socks, and to avoid going barefoot. They were also instructed to notify the staff immediately if they noticed deviation from the 'norm'. Based on the patients' knowledge level and need, additional educational material related to diabetes and foot care was delivered and explained in detail.

\section{Statistical analysis}

We compared all incident diabetic hemodialysis patients before the foot check program implementation date who started hemodialysis between January 2004 and December 2007 (preimplementation cohort), and after the foot check program implementation between January 2008 and December 2011 (postimplementation cohort).

We identified a set of demographic, clinical and laboratory parameters potentially associated with amputation. The patients' demographic characteristics were obtained during a baseline period of 3 months. Clinical, laboratory and treatment variables were averaged over the baseline period. Data are presented as mean, SD, and percent, as appropriate. Point estimates and 95\% CIs of the baseline differences between the preimplementation and postimplementation cohorts are presented.

Major lower limb amputation was the primary end point. We compared the number of major amputations before and after the implementation of foot checks. Poisson regression models with offset of exposure time were constructed with number of major amputations as outcome. Multiple amputations per patient were considered. We performed multivariate analyses with adjustment for age, race, gender, ethnicity, vascular access type for hemodialysis, predialysis systolic blood pressure, albumin, phosphorus, cardiac disease, infection, and peripheral artery disease.
We complemented the main analysis by a sensitivity analysis where we excluded patients from the clinics with $<10$ patients in the postimplementation period, and where percent difference in patient with diabetes number between postimplementation and preimplementation period was $<20 \%$.

Data were censored when patients were transferred to a facility outside Fresenius Medical Care North America, switched to peritoneal dialysis, received a kidney transplant, recovery of kidney function, and at the end of the study period (December 2011). Statistical analyses were performed using SAS V.9.3 (SAS Institute, Cary, North Carolina, USA). A two-sided $\mathrm{p}$ value below 0.05 was considered significant.

\section{RESULTS}

A total of 61292 incident patients were included. We studied 35513 patients in the preimplementation cohort and 25779 patients in the postimplementation cohort. Baseline demographic characteristics, frequency of coexisting conditions, and laboratory parameters are described in table 1 .

There were some statistically significant imbalances between the groups with respect to age, gender, ethnicity, and race, none of which are deemed to be of clinical significance. Notably, however, patients in the postimplementation cohort had a higher comorbidity burden as indicated by a higher rate of hypertension, congestive heart failure, ischemic heart disease, and slightly more cardiac dysrhythmias and peripheral artery disease. Infections were more common in patients without foot checks.

Albeit statistically significant differences were observed for some laboratory and treatment parameters, none of the differences were clinically significant, except possibly for a $0.2 \mathrm{~kg}$ lower interdialytic weight gain and 0.12 units higher $\mathrm{eKt} / \mathrm{V}$ in the postimplementation group.

The implementation of the monthly foot check program was associated with reduced major lower limb amputation. The major lower limb amputation rate was 1.30 per 100 patient years in preimplementation cohort and 1.07 in the postimplementation cohort. Compared with preimplementation cohort, major lower limb amputation decreased by $17 \%$ (Poisson regression; $\mathrm{p}=0.0034$ ).

These findings were corroborated in the multivariate analysis $(p=0.0175)$ with adjustment for age, race, gender, ethnicity, vascular access type for hemodialysis, predialysis systolic blood pressure, albumin, phosphorus, cardiac disease, infection, and peripheral artery disease (table 2).

The sensitivity analysis included data from 8691 patients in the preimplementation period and 8813 patients in the postimplementation period. In the sensitivity analysis, the major lower limb amputation rate differences $(p=0.0083)$ between preimplementation and postimplementation period were even more pronounced (table 3). 
Table 1 Baseline and clinical characteristics of the study cohorts

\begin{tabular}{|c|c|c|c|}
\hline Variable & $\begin{array}{l}\text { Preimplementation } \\
\text { cohort }(n=35513)\end{array}$ & $\begin{array}{l}\text { Postimplementation } \\
\text { cohort }(n=25779)\end{array}$ & Difference $(95 \% \mathrm{Cl})$ \\
\hline Age (years) & $63.1 \pm 12.9$ & $63.3 \pm 12.7$ & $0.23(0.02$ to 0.43$)$ \\
\hline Male sex & $18822(53 \%)$ & $153921(54 \%)$ & 1.0 (1.8 to 0.20$)$ \\
\hline Hispanic ethnicity & $7103(20 \%)$ & $5929(23 \%)$ & 3.0 (3.6 to 2.3$)$ \\
\hline Black race & $11009(31 \%)$ & $7476(29 \%)$ & $-2(-1.26$ to -2.75$)$ \\
\hline White race & $21663(61 \%)$ & $17272(67 \%)$ & $6(6.76$ to 5.24$)$ \\
\hline Body mass index $\left(\mathrm{kg} / \mathrm{m}^{2}\right)$ & $29.4 \pm 8.2$ & $30.1 \pm 8.0$ & 0.77 (0.64 to 0.90$)$ \\
\hline Predialysis systolic blood pressure $(\mathrm{mm} \mathrm{Hg})$ & $150.7 \pm 19.4$ & $150.8 \pm 19.1$ & $0.11(-0.20$ to 0.42$)$ \\
\hline Predialysis diastolic blood pressure $(\mathrm{mm} \mathrm{Hg})$ & $76.0 \pm 11.4$ & $76.3 \pm 11.4$ & $0.34(0.15$ to 0.52$)$ \\
\hline Albumin (g/dL) & $3.47 \pm 0.43$ & $3.50 \pm 0.42$ & $0.02(0.02$ to 0.03$)$ \\
\hline Calcium (mg/dL) & $8.69 \pm 0.61$ & $8.68 \pm 0.60$ & $-0.01(-0.02$ to 0.00$)$ \\
\hline Phosphorus (mg/dL) & $4.92 \pm 1.18$ & $4.92 \pm 1.10$ & $-0.01(-0.03$ to 0.01$)$ \\
\hline Hemoglobin (g/dL) & $11.7 \pm 1.2$ & $11.3 \pm 1.1$ & $-0.41(-0.43$ to -0.39$)$ \\
\hline Serum total cholesterol (mg/dL) & $154.6 \pm 45.8$ & $149.7 \pm 45.4$ & $-4.87(-5.73$ to -4.01$)$ \\
\hline Central venous catheter & $26280(74 \%)$ & $18560(72 \%)$ & $-2.0(-1.28$ to -2.70$)$ \\
\hline Interdialytic weight gain (kg) & $2.46 \pm 0.92$ & $2.25 \pm 0.87$ & $-0.21(-0.22$ to -0.20$)$ \\
\hline Equilibrated Kt/V & $1.35 \pm 0.34$ & $1.47 \pm 0.38$ & $0.12(0.12$ to 0.13$)$ \\
\hline Hemodialysis treatment time (minutes) & $218.7 \pm 23.1$ & $223.3 \pm 21.7$ & 5.54 (5.18 to 5.90$)$ \\
\hline enPCR (g/kg BW/day) & $0.77 \pm 0.22$ & $0.81 \pm 0.24$ & 0.04 (0.04 to 0.04$)$ \\
\hline Peripheral artery disease & $5682(16 \%)$ & $4382(17 \%)$ & $1.00(1.61$ to 040$)$ \\
\hline Congestive heart failure & $10654(30 \%)$ & 8507 (33\%) & 2.99 (3.76 to 2.26$)$ \\
\hline Cardiac dysrhythmias & $3551(10 \%)$ & $2836(11 \%)$ & $1.00(1.49$ to 0.51$)$ \\
\hline Hypertension & $22018(62 \%)$ & $18561(72 \%)$ & 10.00 (10.75 to 9.26$)$ \\
\hline Ischemic heart disease & $9233(26 \%)$ & $7218(28 \%)$ & $2.00(2.71$ to 1.29$)$ \\
\hline Myocardial infarction & $1421(4 \%)$ & 1031 (4\%) & $0.00(0.32$ to -0.31$)$ \\
\hline Infection & $3196(9 \%)$ & $2062(8 \%)$ & $-1.00(-0.55$ to -1.45$)$ \\
\hline
\end{tabular}

$\mathrm{BW}$, body weight; enPCR, equilibrated normalized protein catabolic rate.

\section{DISCUSSION}

We studied 61292 incident diabetic hemodialysis patients to explore whether implementation of a monthly foot check program was associated with lower rate of major limb amputations. The key finding from this observational research was that implementation of foot checks was associated with a significantly lower rate of major lower limb amputations when compared with the preimplementation period. These results were corroborated in a sensitivity analysis.

The observational nature of our study precludes any statements about causality, and the question of whether foot checks independently contribute to better outcome or are mere markers of less disease severity and better

Table 2 Poisson model estimates relating independent variables to amputation counts in the study cohorts

\begin{tabular}{|c|c|c|c|c|}
\hline \multirow{2}{*}{$\begin{array}{l}\text { Independent variable } \\
\text { (Intercept) }\end{array}$} & \multirow{2}{*}{$\begin{array}{c}\text { Estimate } \\
-1.7973\end{array}$} & \multicolumn{2}{|c|}{ Wald $95 \%$ confidence limits } & \multirow{2}{*}{$\begin{array}{l}\text { p Value } \\
<0.0001\end{array}$} \\
\hline & & -2.6318 & -0.9629 & \\
\hline Age (years) & 0.0098 & 0.0045 & 0.0151 & 0.0003 \\
\hline Male sex & 0.189 & 0.0649 & 0.3132 & 0.0028 \\
\hline White race & -0.1182 & -0.2503 & 0.0139 & 0.0795 \\
\hline Hispanic ethnicity & -0.2051 & -0.3711 & -0.0392 & 0.0154 \\
\hline Central venous catheter & 0.1729 & 0.0298 & 0.316 & 0.0179 \\
\hline Predialysis systolic blood pressure $(\mathrm{mm} \mathrm{Hg})$ & -0.0045 & -0.0078 & -0.0011 & 0.0092 \\
\hline Albumin (g/dL) & -0.8923 & -1.0222 & -0.7623 & $<0.0001$ \\
\hline Phosphorus (mg/dL) & 0.0508 & -0.0045 & 0.1061 & 0.0716 \\
\hline Peripheral artery disease & 1.0753 & 0.9454 & 1.2052 & $<0.0001$ \\
\hline Infection & 0.1254 & -0.0561 & 0.3069 & 0.1758 \\
\hline Hypertension & -0.2773 & -0.4089 & -0.1458 & $<0.0001$ \\
\hline Myocardial infarction & -0.0133 & -0.2783 & 0.2516 & 0.9214 \\
\hline Ischemic heart disease & 0.2166 & 0.077 & 0.3562 & 0.0024 \\
\hline Cardiac dysrhythmias & -0.059 & -0.246 & 0.128 & 0.5363 \\
\hline Congestive heart failure & -0.0296 & -0.1624 & 0.1031 & 0.6616 \\
\hline Foot check implementation & -0.15 & -0.2737 & -0.0263 & 0.0175 \\
\hline
\end{tabular}


Table 3 Results of the sensitivity analysis

\begin{tabular}{|c|c|c|c|c|}
\hline \multirow{2}{*}{$\begin{array}{l}\text { Independent variable } \\
\text { (Intercept) }\end{array}$} & \multirow{2}{*}{$\begin{array}{l}\text { Estimate } \\
-1.4139\end{array}$} & \multicolumn{2}{|c|}{ Wald $95 \%$ confidence limits } & \multirow{2}{*}{$\begin{array}{r}\text { p Value } \\
0.0679\end{array}$} \\
\hline & & 2.9316 & 0.1038 & \\
\hline Age (years) & 0.0053 & 0.0044 & 0.015 & 0.2868 \\
\hline Male sex & 0.0628 & 0.1617 & 0.2873 & 0.5837 \\
\hline White race & -0.2072 & 0.4446 & 0.0303 & 0.0872 \\
\hline Hispanic ethnicity & -0.265 & 0.5614 & -0.0313 & 0.0796 \\
\hline Central venous catheter & 0.22 & 0.0418 & 0.4817 & 0.0996 \\
\hline Predialysis systolic blood pressure $(\mathrm{mm} \mathrm{Hg})$ & -0.0063 & 0.0125 & -0.0002 & 0.0436 \\
\hline Albumin $(\mathrm{g} / \mathrm{dL})$ & -0.8414 & 1.0774 & 0.6054 & $<0.0001$ \\
\hline Phosphorus (mg/dL) & 0.1128 & 0.0137 & 0.2119 & 0.0256 \\
\hline Peripheral artery disease & 0.9691 & 0.7331 & 1.2051 & $<0.0001$ \\
\hline Infection & 0.111 & 0.2113 & 0.4334 & 0.4996 \\
\hline Hypertension & -0.3976 & 0.6383 & -0.1568 & 0.0012 \\
\hline Myocardial infarction & -0.2773 & 0.7612 & 0.2066 & 0.2613 \\
\hline Ischemic heart disease & 0.3688 & 0.1194 & 0.6182 & 0.0038 \\
\hline Cardiac dysrhythmias & -0.0479 & -0.38 & 0.2842 & 0.7774 \\
\hline Congestive heart failure & -0.0275 & 0.2656 & 0.2106 & 0.8209 \\
\hline Foot check implementation & -0.2993 & 0.5214 & -0.0772 & 0.0083 \\
\hline
\end{tabular}

medical care cannot be answered based on our analysis. While the association of foot checks with less major amputation was corroborated by multivariate analysis, unmeasured confounders may contribute to the results of this observational study. Of note, the same dialysis clinics were studied in the preimplementation as well as in the postimplementation periods, rendering differences in practice patterns less likely. The reduction of lower limb amputation is important not only because of the devastating nature of lower limb amputations, but also because of its adverse association with mortality. The relation between lower limb amputation and poor survival was indicated in previous research in diabetic dialysis patients showing that 30 days postamputation survival was $88.9 \%$, at 1 year $49.3 \%$, and at 2 years $32.7 \%$; in other words, two-thirds of patients with diabetes died within 2 years after lower limb amputation. ${ }^{1}$ Additionally, the rate of lower limb amputation in patients with diabetes appears to be higher after dialysis initiation. ${ }^{1}$ The incidence of major amputation approached $6 \%$ in the first half year on dialysis, with inadequate hemodialysis being a risk factor. ${ }^{16}$ In a small group mostly of Mäori patients with diabetes, the time between dialysis initiation (hemodialysis or peritoneal dialysis) and amputation was $<1$ year, and $50 \%$ of patients undergoing amputation died within 1 year. ${ }^{11}$

The pathogenesis of lower limb amputation in diabetes is multifactorial. The initial lesion is often caused by minor local trauma, which leads to ulceration. Limb ischemia and peripheral neuropathy have both been implicated in the progression of limb ulcers. ${ }^{17-19}$ Hill et $a \vec{p}$ identified dialysis vintage as a significant predictor of foot complications. Additional risk factors are macroangiopathy and microangiopathy, and extensive atherosclerotic vascular disease, and, possibly, other pathologies related to chronic kidney disease, such as altered calcium/phosphate metabolism. ${ }^{3}$ Dialysis was associated with a two times higher risk of prior amputation, prior foot ulceration, diabetic polyneuropathy, and peripheral arterial disease. This strong association between prevalent foot ulceration and dialysis therapy remained significant after adjusting for potential confounders such as use of footwear, duration of diabetes, neuropathy, and peripheral arterial disease. ${ }^{2}$ A close relationship was also revealed between the incidence of foot ulceration and the start of dialysis in patients with diabetes. ${ }^{16}$

Recently, a systematic review of 30 non-randomized studies quantified major risk factors associated with serious foot ulceration and lower extremity amputation in $>48000$ patients treated with dialysis. ${ }^{20}$ Among other factors, they found that dialysis patients most at risk for foot ulceration were those with previous foot ulceration, peripheral arterial disease and coronary artery disease, and most at risk for amputation were those with previous foot ulceration, peripheral arterial disease and peripheral neuropathy. In this review, dialysis patients with diabetes mellitus were more than three times as likely to have a current foot ulcer and more than seven times as likely to have an amputation. Both complications were more common in people with longer duration of diabetes. ${ }^{20}$ Several studies investigating the prevention of foot ulceration and amputation in patients with diabetes among the general population showed that multidisciplinary foot care improved ulcer healing and reduced amputation rates. ${ }^{21} 22$ In a study of 145 patients, 56 received multidisciplinary foot care (intervention), and 89 received standard care. Significantly fewer recurrent ulcers were seen in the intervention group during a 2-year follow-up. ${ }^{21}$ The multidisciplinary approach included diabetologists, rehabilitation physicians, orthopedic surgeons, podiatrists, and shoemakers, while the standard care team consisted of diabetologist and nurse only. ${ }^{22}$ Larsson showed an almost $50 \%$ reduction in 
amputations over 11 years in Swedish patients with diabetes who were managed by a multidisciplinary approach. ${ }^{23}$ Besides the study by McGrath and Curran ${ }^{11}$ from New Zealand demonstrating lower amputations after instituting an intensive podiatric and orthotic service in Mäori patient with diabetes on hemodialysis, and the study by Rith-Najarian and Gohdes ${ }^{13}$ showing reduced amputation rates in diabetic Native Americans on dialysis when extending primary clinical pathways (called Staged Diabetes Management) to the local dialysis facility for patients with severe ulcers, there are-to the best of our knowledge-only two additional reports demonstrating benefits of prevention of lower limb ulceration and amputation in patients with diabetes with end-stage renal disease. A study in renal transplant recipients has shown the efficacy and importance of intervention to prevent amputation among high-risk patients. In that study, a special foot clinic for patients with diabetes with renal transplant reduced digital gangrene and major amputations, led to a higher healing rate of foot ulceration, and only two major amputations in 50 patients over 4 years. ${ }^{24}$ A study on 132 diabetic peritoneal dialysis patients indicated that a chiropody program decreased amputation rates. The intervention included foot care education, and assessment and treatment by chiropodists. Predictors of time to amputation or death were age, peripheral and cerebrovascular disease, and chiropodist intervention. ${ }^{25}$ Patients with diabetes with end-stage renal disease are at a high risk of lower limb amputation, and dialysis is independently associated with foot ulceration. ${ }^{1-4} \quad 8 \quad 16 \quad 18 \quad 19 \quad 26$ Consequently, patients with diabetes on chronic hemodialysis require intensive foot care. There is agreement that these highrisk patients should be actively targeted for prevention of ulceration. ${ }^{101819}$ However, the busy treatment schedule requiring thrice weekly hemodialysis visits may actually present a barrier to accessing good foot care. Only $30 \%$ of hemodialysis patients who presented with one or more risk factors for foot ulcerations had attended a podiatrist in 12 months. ${ }^{18}$ On the other hand, dialysis patients have interactions with nephrology nurses on a regular basis. This relationship between nurse and patient could serve as a basis for preventive and self-care practices, and foot care can be integrated in the regular workflow of a dialysis facility. ${ }^{17}{ }^{18}$ Therefore, optimising foot care with routine monthly intradialytic foot checks was implemented at Fresenius Medical Care North America facilities in 2008. Since then, the goal has been for all patients with diabetes to receive routine foot care once a month while attending hemodialysis. The program recommends that patients with foot ulceration be referred to a specialist multidisciplinary foot clinic for further assessment and management according to American Diabetes Association guidelines. ${ }^{27}$

\section{Strengths and limitations of this study}

Our study has limitations-first and foremost its observational nature. However, ethical considerations render a randomized controlled trial of foot checks questionable. Second, our research would be enhanced if we included data on the number of referrals to wound care, podiatry and orthopedics before and after implementation of foot care; unfortunately, this information is not captured in the current data base. Third, a cost-benefit analysis would have been important. Owing to the retrospective nature of our study, key determinants required for costbenefit analysis were not captured, for example, on the cost side, staff time required for foot checks, supplies utilized, where and how ensuing procedures happened, and the equipment and supplies used during those procedures. In addition, the benefit side of this interventional is even more difficult to quantify. We strongly believe that cost-benefit analysis should be the focus of carefully designed future studies. Fourth, our study is limited to observations made in our dialysis network. However, we believe that the large number of patients treated in facilities across the USA favor the generalizability of the results. In addition to the aforementioned diverse population, another strength of our study is that the foot checks deployed are highly standardized and protocol-driven, making local variations unlikely. Organization of care in dialysis facilities for patients with diabetes with end-stage renal disease could definitely have an impact on the foot disease. On the other hand, the prevalence of peripheral arterial disease is greater in these patients but is often underdiagnosed and undertreated. ${ }^{10}$ The 2010 KDIGO guidelines recommend screening at the time of dialysis initiation, ${ }^{15}$ but detection of peripheral arterial disease is problematic due to lack of clarity regarding classic clinical symptoms and diagnostic testing. ${ }^{28}$ Implementation of a routine foot check is one intervention to reduce the devastating complication of peripheral arterial disease. A next step for future intervention to improve patient-centered outcomes should be to prevent peripheral arterial disease from developing in this very high risk group of patients. ${ }^{28}$ Despite the encouraging results of our intervention study, there is still a need for further, more effective methods in preventing peripheral arterial disease from developing in renal patients. ${ }^{15}$

\section{Conclusions}

Monthly foot checks are associated with reduction of major lower limb amputations in incident diabetic hemodialysis patients. Implementation of foot checks during dialysis may have the potential to reduce amputations and morbidity, and improve quality of life.

Contributors AMP wrote the manuscript and interpreted the data. LU analyzed the data and conducted statistical analyses. LS collected the data. VP, JR, FWM and EL contributed to the discussion and reviewed the manuscript. PK edited the manuscript and researched the data. All the authors read and approved the final version of the manuscript. PK is the guarantor of this work and, as such, had full access to all the data in the study and takes responsibility for the integrity of the data and the accuracy of the data analysis. 
Funding Renal Research Institute.

Competing interests FWM and PK hold stock in Fresenius Medical Care. JR and EL were full time employees of Fresenius Medical Care at the time this study was conducted; they left the company in September 2013 and May 2015, respectively.

Ethics approval This observational study was approved by the Institutional Review Board of Beth Israel Medical Center, New York.

Provenance and peer review Not commissioned; externally peer reviewed.

Data sharing statement No additional data are available.

Open Access This is an Open Access article distributed in accordance with the Creative Commons Attribution Non Commercial (CC BY-NC 4.0) license, which permits others to distribute, remix, adapt, build upon this work noncommercially, and license their derivative works on different terms, provided the original work is properly cited and the use is non-commercial. See: http:// creativecommons.org/licenses/by-nc/4.0/

\section{REFERENCES}

1. Eggers PW, Gohdes D, Pugh J. Nontraumatic lower extremity amputations in the Medicare end-stage renal disease population. Kidney Int 1999;56:1524-33.

2. Ndip A, Lavery LA, Lafontaine $\mathrm{J}$, et al. High levels of foot ulceration and amputation risk in a multiracial cohort of diabetic patients on dialysis therapy. Diabetes Care 2010;33:878-80.

3. Hill MN, Feldman HI, Hilton SC, et al. Risk of foot complications in long-term diabetic patients with and without ESRD: a preliminary study. ANNA J 1996;23:381-6; discussion 387-8.

4. Ndip A, Rutter MK, Vileikyte L, et al. Dialysis treatment is an independent risk factor for foot ulceration in patients with diabetes and stage 4 or 5 chronic kidney disease. Diabetes Care 2010;33:1811-16.

5. Lavery LA, Lavery DC, Hunt NA, et al. Amputations and foot-related hospitalisations disproportionately affect dialysis patients. Int Wound $\mathrm{J}$ 2015;12:523-6.

6. Pecoraro RE, Reiber GE, Burgess EM. Pathways to diabetic limb amputation: basis for prevention. Diabetes Care 1990;13:513-21.

7. Mark P, McNally M, Jones GC. Deficiencies in foot care of diabetic patients on renal replacement therapy. Practical Diabetes Int 2003;20:294-6.

8. O'Hare AM, Bacchetti $P$, Segal M, et al. Factors associated with future amputation among patients undergoing hemodialysis: results from the Dialysis Morbidity and Mortality Study Waves 3 and 4. Am J Kidney Dis 2003;41:162-70.

9. Schomig M, Ritz E, Standl E, et al. The diabetic foot in the dialyzed patient. J Am Soc Nephrol 2000;11:1153-9.

10. Al Thani H, El-Menyar A, Hussein A, et al. Prevalence, predictors, and impact of peripheral arterial disease in hemodialysis patients: a cohort study with a 3-year follow-up. Angiology 2013;64:98-104.

11. McGrath NM, Curran BA. Recent commencement of dialysis is a risk factor for lower-extremity amputation in a high-risk diabetic population. Diabetes Care 2000;23:432-3.
12. Rith-Najarian S, Branchaud C, Beaulieu O, et al. Reducing lower-extremity amputations due to diabetes. Application of the staged diabetes management approach in a primary care setting J Fam Pract 1998:47:127-32.

13. Rith-Najarian S, Gohdes D. Preventing amputations among patients with diabetes on dialysis. Diabetes Care 2000;23:1445-6.

14. National Kidney Foundation. K/DOQI clinical practice guidelines for chronic kidney disease: evaluation, classification, and stratification. Am J Kidney Dis 2002;39(2 Suppl 1):S1-266.

15. Herzog CA, Asinger RW, Berger AK, et al. Cardiovascular disease in chronic kidney disease. A clinical update from Kidney Disease: Improving Global Outcomes (KDIGO). Kidney Int 2011:80:572-86.

16. Game FL, Chipchase SY, Hubbard R, et al. Temporal association between the incidence of foot ulceration and the start of dialysis in diabetes mellitus. Nephrol Dial Transplant 2006;21:3207-10.

17. Benhamou Y, Edet S, Begarin L, et al. Transcutaneous oxymetry as predictive test of peripheral vascular revascularization in haemodialysis population. Nephrol Dial Transplant 2012;27: 2066-9.

18. Kaminski M, Frescos N, Tucker S. Prevalence of risk factors for foot ulceration in patients with end-stage renal disease on haemodialysis. Intern Med J 2012;42:e120-8.

19. Ndip A, Lavery LA, Boulton AJ. Diabetic foot disease in people with advanced nephropathy and those on renal dialysis. Curr Diab Rep 2010;10:283-90.

20. Kaminski MR, Raspovic A, McMahon LP, et al. Risk factors for foot ulceration and lower extremity amputation in adults with end-stage renal disease on dialysis: a systematic review and meta-analysis. Nephrol Dial Transplant 2015;30:1747-66.

21. Dargis V, Pantelejeva O, Jonushaite A, et al. Benefits of a multidisciplinary approach in the management of recurrent diabetic foot ulceration in Lithuania: a prospective study. Diabetes Care 1999;22:1428-31.

22. Speckman RA, Frankenfield DL, Roman $\mathrm{SH}$, et al. Diabetes is the strongest risk factor for lower-extremity amputation in new hemodialysis patients. Diabetes Care 2004;27:2198-203.

23. Larsson J, Eneroth M, Apelqvist J, et al. Sustained reduction in major amputations in diabetic patients: 628 amputations in 461 patients in a defined population over a 20 -year period. Acta Orthop 2008;79:665-73.

24. Foster AV, Snowden S, Grenfell A, et al. Reduction of gangrene and amputations in diabetic renal transplant patients: the role of a special foot clinic. Diabet Med 1995;12:632-5.

25. Lipscombe J, Jassal SV, Bailey S, et al. Chiropody may prevent amputations in diabetic patients on peritoneal dialysis. Perit Dial Int 2003:23:255-9.

26. Jones NJ, Chess J, Cawley S, et al. Prevalence of risk factors for foot ulceration in a general haemodialysis population. Int Wound $J$ 2013;10:683-8.

27. American Diabetes Association. Standards of medical care in diabetes-2008. Diabetes Care 2008;31(Suppl 1):S12-54.

28. Garimella PS, Hart PD, O'hare A, et al. Peripheral artery disease and CKD: a focus on peripheral artery disease as a critical component of CKD care. Am J Kidney Dis 2012;60: 641-54. 